\section{Pending questions in venous ulcers management. Report from a Symposium of the World Union of Wound Healing Societies International Congress}

\author{
Giorgio Guarnera, ${ }^{1}$ Paolo Zamboni, ${ }^{2}$ \\ Olle Nelzen, ${ }^{3}$ Ferdinando Mannello, ${ }^{4}$ \\ Anneke Andriessen ${ }^{5}$
}

${ }^{1}$ Department of Vascular Surgery, Aurelia Hospital, Rome, Italy; ${ }^{2}$ Vascular Diseases Center, University of Ferrara, Italy; ${ }^{3}$ Vascular Surgery Departments of Research and Development and Vascular Surgery, Skaraborg Hospital Skövde, Sweden; ${ }^{4}$ Section of Clinical Biochemistry and Molecular Genetics, Department of Biomolecular Sciences, University Carlo Bo, Urbino, Italy; ${ }^{5}$ Radboud UMC, Nijmegen \& Andriessen Consultants, Malden, The Netherlands

\begin{abstract}
Venous ulcers represent the most frequent ulcers and have a high clinical and socioeconomic significance. Nevertheless, pending questions remain on epidemiology, genetic and biomolecular mechanisms, contraindications and risk factors of compression therapy, role of pharmacological therapy. Overall prevalence in the range of $1-2 \%$ and point prevalence of $0.08-0.6 \%$ can be reduced if risk factors are identified and treated early. In this context, analysis of factor XIII and hemocromatosis gene polymorphisms, with the C282Y and H63D variants, may be of great importance. Also MMP12 gene polymorphism and the imbalance between matrix metalloproteinases and their tissue inhibitors are able to impair wound healing via deleterious degradation process of extra-cellular matrix. From a therapeutic point of view, conflicting recommendations exist on relative contraindications, risks and adverse events of compression therapy. Some studies showed that the administration of pentoxyphilline, Micronized purified flavonoid fraction (MPFF) and sulodexide was effective in accelerating ulcer healing, but there is no data on the duration of treatment, recurrence rates and cost-effectiveness relationship.
\end{abstract}

\section{Introduction}

During World Union of Wound Healing Societies International Congress (25-29 of September 2016, Florence, Italy) a Scientific Session was held on venous ulcers with the aim of discussing controversies in this pathology management.

Venous ulcers are the most frequent leg ulcers. Clinical pathways for patients with venous leg ulcers should include accurate diagnosis and the use of appropriate diagnostic and therapeutic tools. Nevertheless there are many open issues in terms of epidemiology, pathophysiology and treatment.

Willingly, we did not deal with wellknown topics such as superficial venous reflux abolition (ESCHAR study underlined the importance of surgery in preventing recurrences) ${ }^{1}$ or venous ulcers local therapy. On the contrary, we have chosen current topics with the objective of draw attention on controversial issues in venous ulcers.

The article presents ideas, experiences and suggestions offered by known Specialists in different medical branches, in order to answer provocative questions on pending issues in venous ulcers management. The aim of the paper is to identify gaps in the evidence and methodological deficiencies and assess future research needs for chronic venous ulcer care.

\section{What is the real prevalence of venous ulcers?}

There are very few epidemiological studies to generate data from and most of them are 10-30 years old. There are no newer estimates available. There have been several epidemiological studies performed reporting on leg ulcer point prevalence, but without properly defining the causes of the ulcers. ${ }^{2}$ We often talk about leg ulcers as being venous in a majority of cases when in fact the true point prevalence is largely unknown. Only very few have determined the etiology by means of objective assessment tools, such as color Doppler ultrasound (CDU) or plethysmography. In a Swedish study in Skaraborg detailed assessment showed that only half were of pure venous origin, at that time a point prevalence of $0.16 \%$. $^{3}$ There are several ways of looking at the various forms of prevalence, point, period and overall (Table 1). It is important to realize that they all mean different things. Point prevalence is different from period prevalence and overall or lifetime prevalence and they should not be mixed up. We are mostly talking about point prevalence expressing how many are hav-
Correspondence: Giorgio Guarnera, Department of Vascular Surgery, Aurelia Hospital, Rome, Italy.

E-mail: gguarnera@tiscali.it

Key words: Epidemiology; gene polymorphisms; biomarkers; compression therapy; pharmacological therapy.

Conflict of interests: the authors declare no conflict of interests.

Received for publication: 23 June 2020.

Revision received: 27 Septmber 2020.

Accepted for publication: 30 September 2020 .

This work is licensed under a Creative Commons Attribution 4.0 License (by-nc 4.0).

(C) Copyright: the Author(s), 2020

Licensee PAGEPress, Italy

Veins and Lymphatics 2020; 9:9206

doi:10.4081/vl.2020.9206

ing active ulcers at any one point of time or how many that will ever get a venous ulcer (overall prevalence). Another problem in assessing prevalence is that most studies have based their estimates on patients known to and taken care of within the health care system. In addition to that there are people taking care of their ulcers by themselves and that can only be assessed by performing random samples studies based on the entire population. The true prevalence is therefore higher than what is registered within the health care system.

Incidence is yet another measurement usually meaning how many new cases (first time) that will be expected during one year (Table 1). It seems that around one out of ten patients with venous ulcer has a first time ulcer with a duration of less than one year. ${ }^{3}$ The yearly incidence has been estimated to be in the range of $10-30 / 100,000$ population in the western world. ${ }^{4}$ The figure of $1 \%$ having ever had a venous ulcer is probably a fairly correct estimate since it has been reproduced in several studies. ${ }^{4}$ Similar levels were found in the two most recent population studies on venous disease from the Bonn vein study in Germany ${ }^{5}$ and the Edinburgh vein study in Scotland. ${ }^{6}$ The point prevalence may vary a lot depending on how actively CDU has been used in ulcer diagnosis and to what extent surgical intervention have been used to eradicate superficial venous incompetence. It is important to realize that also patients that heal readily following compression treatment benefit from surgical intervention due to its superior effectiveness in preventing recurrent ulceration. ${ }^{7,8}$ Without early surgical inter- 
vention in these cases point prevalence is likely to stay high or even increase. There are no data showing that conservative management can reduce the point prevalence of venous ulcer and most experts are expecting an increasing number of patients with venous ulcer due to ageing populations. ${ }^{9}$

This results in a wide range for point prevalence estimates of active venous ulcer from $0.08 \%$ up to $0.6 \%{ }^{3}$ There are more available and stable data regarding overall or lifetime prevalence of venous ulceration that can be expected to be in the range of 1$2 \%$ and to lower that figure is more difficult and requires wide use of durable varicose vein treatments in our populations throughout many decades. Recurrence is lowered effectively by surgery as opposed to conservative compression alone. ${ }^{1}$ Varicose vein surgery and also never endovenous techniques to ablate saphenous vein incompetence have been shown effective in preventing recurrence and also (since this presentation was given) duplicate improve ulcer healing. ${ }^{10}$ In Skaraborg, Sweden, point prevalence was followed closely by using objective diagnostics and repeated epidemiological cross-sectional studies performed 2002 and 2014. ${ }^{11,12}$ These studies allowed us to monitor the effect of management and treatment changes to improve the outcomes. Per preliminary data the point prevalence has been reduced by some $70 \%$ from 1988 , point prevalence 0.16 and down to $0.05 \%$ in 2014..$^{11,12}$

Increased use of early surgical intervention based on proper diagnosis with CDU is likely to halt and reduce the feared increase of venous ulceration in the world.

\section{Is there a genetic predisposition to venous leg ulcers?}

We concentrated our attention to three gene families, based on notions of pathophysiology which candidate the followings: i) iron trafficking genes. In chronic venous insufficiency the iron overload in the tissue is visible at naked eye. Activation of Fenton reaction with production of free radicals and, in turn, matrix metalloproteinases (MMPs) activation are pathways deeply investigated in venous leg ulceration; ${ }^{13-17}$ ii) factor XIII polymorphysms. Activated fac- tor XIII (FXIIIa) is responsible for the cross-linking of fibrin monomers during the stabilization process of thrombus, ${ }^{18}$ and we basically know it as a key element of the hemostatic process. To the contrary, although less reported, FXIIIa plays a pivotal role in the stabilization of the provisional extra-cellular matrix (ECM) during any healing process. FXIIIa is also responsible in shuttling the provisional matrix towards the definitive one. Because the polymorphisms of Factor XIII are numerous, frequent, and capable to change the molecular properties they were investigated by our group; ${ }^{18-20}$ iii) MMPs. It has been demonstrated, in course of severe venous stasis and/or venous leg ulcers an elevated gene expression for several families of MMPs and a reduced one for tissue inhibitor of MMPs (TIMPs). Such unrestricted MMPs proteolytic activity is commonly considered the final executioner of a pathogenetic chain leading to matrix disruption and ulcer development. ${ }^{18-19}$ This event together with the interstitial migration of macrophages have been proven to be fundamental component of the inflammatory cascade activated in the matrix in course of chronic venous disease (CVD). ${ }^{21,22}$ Now this appears at the beginning of the paragraph describing the genetics of venous leg ulcers (VLU). Although the number of factors involved in the etiology of chronic wounds is extremely high, a recognition of functional gene variants significantly involved in wound healing and venous ulcer establishment, may help our prognosis, diagnosis and treatment capabilities of chronic wounds. Over the years, we can read rigorous analyses of FXIII and mutated hemochromatosis protein [human factors engineering $(H F E)]$ gene polymorphism and their prognostic implication in precocious ulcer onset and in healing time after superficial venous surgery. ${ }^{18-20,23} \mathrm{HFE}$ gene polymorphisms, with the $\mathrm{C} 282 \mathrm{Y}$ and $\mathrm{H} 63 \mathrm{D}$ variants, are the most commonly recognized genetic defects in iron metabolism, and they have a vital role in predicting ulcer development. Since the iron hypothesis does not explain why leg iron deposits in CVD produce lesions only in some individuals, it was hypothesized that such individual differences could be genetically determined on the basis of HFE gene mutations, which are associated with an increased iron efflux from the macrophage. ${ }^{14,15,24,25}$ An example of the gene-environment interaction is reflected by an increased risk of 6- to 7-fold for VLU onset when there coexists an overlapping condition of CVD and C282Y carrier condition. ${ }^{26}$ Similarly, gene variant analysis of FXIII had given an important clinical conclusion in predicting clinical phenotypes to investigate the healing time and ulcer size in chronic wounds. ${ }^{27}$ Val34Leu polymorphism, strictly associated to the thrombin activation site, is the main functional polymorphism influencing FXIII activation with its approved role in increasing the catalytic efficiency of the molecule and the rate of fibrin clot stabilization with potent capacity to alter the structure of fibrin clot. Higher plasma FXIII activity, because of Val34Leu variant, may link more a2-antiplasmin to fibrin and thus causes the formation of more rigid and tougher fibrin gel that is chemically/mechanically more stable as well as resistant to fibrinolysis, mitigating the plasmin-dependent activation process of pro-MMPs by direct plasminantiplasmin inhibition. An earlier-activated FXIII molecule becomes more available to cross-link ECM components, stabilizing them against wasteful proteolysis. ${ }^{18-20,23}$

In a successive cohort of CVU subjects, we investigated if there was some relation between FXIII variants (Val34Leu, Pro564Leu, Tyr204Phe, His95Arg) and venous ulcer surface. After genotyping of cases and controls, the statistical analysis showed no differences in genotype frequencies for all polymorphisms studied, thus excluding any risk role in CVU establishment ascribable to these FXIII gene variants. The main finding of this study was the protective role of Leu34 and Leu564 polymorphisms in the whole group and primary CVU, whereas in the post thrombotic cases it did not show any protective role. The area of the ulcer was inversely related with the presence of the Leu34 and Leu564 allele in an independent fashion and without synergistic effects (hazard ratio, $4.14 ; 95 \%$ confidence interval, 2.1 to 8.2 ; Leu $34 \mathrm{P}$ value $=0.00005$; Leu564: $P$ value $=0.00005$ ). In this view these 2 variants of FXIIIA can be considered as modulators in lesion progression and extension. ${ }^{18}$ Also the role of MMP gene variants was suspected in clinical manifestation of CVD. ${ }^{22,23}$ Recently, we investigated single nucleotide polymor-

Table 1. Terminology of ulcers epidemiology (World Health Organization) ${ }^{13}$

\begin{tabular}{ll}
\hline Incidence & Number of new cases usually per year and in relation to the population \\
Point prevalence & Proportion of people with open ulcers at a certain short time frame (1-2 months) \\
\hline Period prevalence & Proportion of people with open ulcers during a longer time frame (usually 1 year) \\
Overall prevalence & All people who have ever suffered from an ulcer (open + healed; lifetime prevalence) \\
\hline
\end{tabular}


phism (SNPs) in the promoter region of the MMP12 gene (MMP12; -82AG) that might have a role in leg ulcer progression as well in other complex diseases via ECM degradation. Concerning the role of our previous SNPs as prognostic markers in CVD, we propose MMP12 gene polymorphism ($82 A G)$ as a putative prognostic marker in VLU progression. ${ }^{27}$ The immediate clinical application of gene variant analysis and evaluation implies that the high-risk minority of patients could be identified in advance. We think that a simple blood test could act as a genetic screening device. Based on the data shown above, prevention program of VLU in primary varicose veins could be extraordinarily improved by using genetic markers in our initial patient assessment.

Table 2. Inflammatory and proteolytic biomarkers in chronic wound fluids from inflammatory and granulating venous leg ulcers.

Venous leg ulcer
Inflammatory

\begin{tabular}{cc}
$\uparrow$ IL-1 & \\
$\uparrow$ IL-12 & \\
$\uparrow$ IL-10 & $\uparrow$ IP-10 \\
$\uparrow$ IL-8/CXCL8 & $\uparrow$ RANTES \\
$\uparrow$ GM-CSF & $\uparrow$ PDGF-BB \\
$\uparrow$ VEGF & $\uparrow$ MMP-1 \\
$\uparrow$ MMP-2 & $\uparrow$ MMP-7 \\
& \\
$\uparrow$ MMP-9 & $\uparrow$ MMP-13 \\
$\uparrow$ MMP-12 & $\uparrow$ TIMP-4 \\
$\uparrow$ TIMP-1 & \\
$\uparrow$ TIMP-2 & \\
\hline
\end{tabular}

IL, interleukin; CXCL8, cytokine also known as interleukin-8; GM-CSF, granulocyte macrophage colony stimulating factor; VEGF, vascular endothelial growth factor; MMP, matrix metalloproteinase; IP-10, interferon gamma-induced protein 10; RANTES, regulated on activation, normal T cell expressed and secreted; PDGF, platelet derived growth factor; TIMP, tissue inhibitor of metalloproteinases.

\section{Biochemical and molecular markers in wound healing}

The discovery of novel genomic, transcriptomic and proteomic biomarkers may significantly help the improvement of risk assessment, prevention strategies and medical decision making in the field of CVD.

A number of evidences suggested that proteins regulating extracellular matrix turnover and remodeling are involved in Chronic venous insufficiency ( CVI ) evolution and progression; 28 in this respect, ECM-degrading proteolytic enzymes, belonging to the matrix metalloproteinase family, have been characterized as key players in the intricate network between inflammatory signals and changes in vascular endothelium and dermal ECM. ${ }^{29-31}$

The imbalance between MMPs and their inhibitors TIMPs is able to impair wound healing via deleterious degradation process of ECM, leading to loss of epidermal integrity characteristic of chronic hardto-heal wounds. ${ }^{32}$ Noteworthy, some MMPs are related to healing conditions in ulcers characterized by granulation tissue, whereas other MMPs are mainly found in inflammatory hard-to-heal wound with chronic condition of inflammation. ${ }^{30}$ The list of proteolytic enzymes in wound microenvironment is reported in Table 2.Recently, it has been demonstrated that in wound fluids collected from chronic venous leg ulcers, proand anti-inflammatory cytokines as well as growth factors expressed in the wound microenvironment may represent useful markers for demonstrating that cytokine dysregulation guide deleterious inflammatory processes of ECM, and identifying chronic hard-to-heal wounds. ${ }^{32}$ Moreover, these biomarkers may represent possible targets for a better therapeutic approach. Noteworthy, some cytokines and growth factors are related to healing conditions in ulcers characterized by granulation tissue, suggesting the optimal therapeutic conditions for improving the healing of CVI ulcers. ${ }^{33}$

The identification of accurate biomarkers in wound microenvironments, the explication of CVI disease mechanisms, the stratification of patients groups according to healing process, and the development of protocols and assay platforms for using clinical biomarkers as diagnostic indicators of healing status may represent a crucial subject for future directions in CVI studies.

This overview highlights the news in venous wound research, suggesting the characterization of known wound biomarkers, the identification of new biomarkers and the future development of new panels of biomarkers for the prediction of chronic wound outcome, in conjunction to possible effective therapeutic potential.

Finally, in order to assess the potential usefulness of the clinical biomarkers in the field of CVI in a way that can be useful for clinicians, we highlight and may suggest that currently genomic biomarkers can only give information (of low to medium risk) of developing clinical conditions related to CVI and CVD, due to multigenic participation in the disease. ${ }^{34}$

For what concerns the proteomic biomarkers (including cytokinome and degradome), the increasing number of studies put the research approach at a good point of developing and are coming to be used in the clinical practice for disease predisposition, identification of healing status and follow-up tracking during pharmacologic treatment.

In the near future the hope is to validate stratification risk, which may integrate data from molecular and biochemical biomarkers in conjunctions to clinical assessment to provide information on CVI early diagnosis, staging and therapeutic efficacy.

Table 3. Contraindications to compression therapy.

\begin{tabular}{|c|c|c|c|c|c|}
\hline Absolute contraindications & $\begin{array}{l}\text { No } \\
\text { ref. }\end{array}$ & Relative contraindications & $\begin{array}{l}\text { No } \\
\text { ref. }\end{array}$ & $\begin{array}{l}\text { Contraindications without } \\
\text { classification }\end{array}$ & $\begin{array}{l}\text { No } \\
\text { ref. }\end{array}$ \\
\hline Arterial occlusive disease & $35,42,43$ & \multicolumn{2}{|l|}{ Diabetes mellitus; peripheral neuropathy } & 47,48 & $\begin{array}{l}\text { Heart } \\
\text { failure }\end{array}$ \\
\hline Heart failure & $35,42,43$ & Heart failure & $35,42,43$ & Neuropathy & 39 \\
\hline $\begin{array}{l}\text { Ankle brachial pressure } \\
\text { index }<0.5-0.8\end{array}$ & $35,42,43,44$ & $\begin{array}{l}\text { Compensated peripheral arterial } \\
\text { occlusive disease }\end{array}$ & $42,43,45$ & & \\
\hline $\begin{array}{l}\text { Extensive thrombophlebitis, } \\
\text { thrombosis or suspected thrombosis }\end{array}$ & 45,46 & $\begin{array}{l}\text { Intolerance to dressing } \\
\text { material/allergies }\end{array}$ & $42,43,45$ & $\begin{array}{l}\text { Extensive thrombophlebitis, } \\
\text { thrombosis or suspected thrombosis }\end{array}$ & $\begin{array}{l}39 \\
\text { is }\end{array}$ \\
\hline Phlegmasia coerulea dolens & 45 & Skin diseases & 43,46 & Erysipelas & 39 \\
\hline Erysipelas & $35,43,44$ & Malignant diseases & 43,47 & Serious non-controlled hypertension & 42,46 \\
\hline Serious non-controlled hypertension & $35,43,44$ & Ankle brachial pressure index $<0.5-0.8$ & 35,42 & & \\
\hline
\end{tabular}




\section{Contraindications, risk factors, adverse events in venous leg ulcer compression therapy}

Currently there is a lack of clarity on contraindications, risk factors, adverse events and complications, when applying compression therapy for venous leg ulcer patients. ${ }^{35}$

A literature review was conducted of current guidelines on venous leg ulcer prevention, management and maintenance (Table $3^{35-48}$ ).

The development of guidelines was done by various disciplines and organizations, including phlebologists, dermatologists, specialized nurses, vascular surgeons and multidisciplinary groups.

Many of the selected guidelines used literature that was more than 10 years old. ${ }^{36}$ As this is a field that is evolving and new technologies are making their way to the market, this may pose issues. ${ }^{36}$

Guidelines agreed on the following absolute contraindications: arterial occlusive disease, heart failure and ankle brachial pressure index $(\mathrm{ABPI})<0.5$, but gave conflicting recommendations on relative contraindications (caution should be used when procedures are used).

Arterial circulation in the lower limbs and clinical implications of ABPI may be defined $^{35,42}$ as follows: normal ABPI $>1.00$ 1.3 , compression therapy can be applied. For those with an ABPI between 0.8-1.0 the presence of mild peripheral disease should be considered and compression can be applied with caution. Significant arterial disease may be present in case of an ABPI $\leq 0.8-0.6$ and only modified compression can be used with caution. Referral to a vascular specialist is recommended in these cases. Critical ischemia is likely to be present in those with an ABPI of $<0.5$, compression is contraindicated and urgent referral to a vascular specialist is needed.

Some guidelines report contraindications without classification in absolute and relative. ${ }^{37,38}$ Extensive thrombophlebitis, thrombosis or suspected thrombosis were recognized as contraindications or absolute contraindications, ${ }^{38,39}$ but the majority of guidelines recommend for patients with proximal deep vein thrombosis to wear graduated compression stockings. ${ }^{40}$

Moreover definitions were unclear and not consistent. As practice in various countries may differ greatly from some of the countries where guidelines were developed, recommendations given may not be achievable. Often guidelines are text heavy and not designed for practical clinical use. Patient focused clinical pathways may be a more practical way forward to optimize care for venous leg ulcer patient management. ${ }^{41}$

Pressure marks, necrosis, friction damage and leg ulcer formation, as a result of poorly applied compression or the wrong type of compression, were identified as risk factors and adverse events in all of the selected guidelines. ${ }^{35-48}$

Complications of compression therapy can almost always be prevented when adequate assessment is performed and clinicians are skilled in applying compression. Effective patient education improves patient's outcome. ${ }^{41}$

\section{Venous ulcers and drugs: an adjuvant therapy?}

In recent years, an increasing number of drugs was widely used in the treatment of the chronic venous disease. Their role in venous ulcers healing remains controversial.

Pentoxifylline was the first drug to be extensively studied: the rationale for its use is based on its anti-inflammatory and hemorheological properties consisting in reducing blood viscosity, decreasing platelet aggregation, antiradical action and reducing cytokine expression..$^{49}$ A Cochrane systematic review of 7 randomized controlled trials (RCTs), involving 659 patients, showed pentoxifylline with compression to be more effective than placebo and compression in term of complete ulcer healing over 8 to 24 weeks ( $64 \%$ of healing vs $40 \%){ }^{50}$

Micronized purified flavonoid fraction (MPFF) has shown to accelerate venous ulcer healing. Of particular interest is its action on inflammatory reactions associated with venous hypertension. MPFF modifies leukocyte-endothelial interactions, inhibiting the expression of intercellular adhesion molecule and vascular adhesion molecule, as well as the expression of leukocyte adhesion molecule on monocytes and neutrophils. $^{51,52}$ In a meta-analysis of five prospective RCTs involving 723 patients with venous ulcers MPFF therapy was compared with compression therapy, alone in three studies, combined with placebo in two studies. Ulcers between 5 and $10 \mathrm{~cm}^{2}$ had a $40 \%$ better chance of healing with MPFF, whereas in venous ulcers aged between 6and 12-months flavonoids increase ulcer healing by $44 \% .{ }^{53}$

Another group of drugs tested in coadjuvant ulcer therapy comprises the glycosaminoglycans (GAGs).

Sulodexide, a sulfated polysaccharide complex extracted from porcine intestinal mucosa, has an antithrombotic action, restores integrity and permeability of endothelial cells, modulates inflammatory pathways in chronic venous disease. ${ }^{54,55}$ Four RCTs, involving 488 patients, demonstrated that oral sulodexide plus compression, compared with compression alone, is more effective at increasing healing rates at 1 to 3 months. ${ }^{56}$

Mesoglycan, composed of highly purified natural GAGs, acts through an enhancement in endogenous fibrinolysis and in antithrombotic properties of plasma. A multi-center double-blind placebo-controlled RCT, involving 183 patients, at 24 weeks showed healing of venous ulcers in $97 \%$ of the mesoglycan group and in $82 \%$ of the placebo group

Methodological shortcomings and bias limit the validity of results from trials involving other drugs.

Trials to determine whether low-dose aspirin therapy may improve time to healing and decrease the number of recurrent venous ulcers are in progress. ${ }^{57}$

If we analyze papers on pharmacological treatment of venous leg ulcers, published in 2016, we can find statements which support the use of pentoxifylline in term of effectiveness and promising evidence. $^{58,59}$

In an open-label, observational, nonparallel trial on 70 patients, the use of sulodexide and diosmin-hesperidin was effective in accelerating ulcer healing, controlling pain and improving lipodermatosclerosis. ${ }^{60} \mathrm{~A}$ Cochrane review, including four trials with a total of 463 participants, showed that sulodexide may increase the healing of venous ulcers, but the evidence is only of low quality. ${ }^{61}$

Vasoactive drugs were proven to be effective in treating symptoms and slow down the progression of chronic venous disease. In venous ulcers management, there are no high-quality clinical trials on phlebotonic drugs which consider pain, quality of life and healing rate as parameters. ${ }^{62,63}$

Pending questions remain. We don't know if the dose of a drug should be the same for varicose veins and for venous ulcers and if a drug has the same efficacy in different hemodynamic patterns. Moreover, there are no recommended limits on the duration of treatment and there is no information on the recurrence rates.

It emerges the need to study serum inflammatory markers to know more about targets of venotonic therapy and to consider pain and quality of life as critical parameters of efficacy. Finally, cost-effectiveness relationship should guide our choices. 


\section{Conclusions}

Venous leg ulcers are characterized by a long healing process and repeated cycles of ulceration. Multiple studies were conducted to identify risk factors for delayed healing and recurrence of venous leg ulcers. ${ }^{64}$

Moreover, many studies were published to determine whether particular dressings or topical agents improve the probability of healing of venous leg ulcers in any care setting and to rank treatments in order of effectiveness, with assessment of uncertainty and evidence quality. ${ }^{64,65}$

Our work aims to draw attention on open issues and controversies regarding venous ulcers management.

We do not propose strict rules, but we offer suggestions and encourage study and research.

There are very few epidemiological studies to generate data from. Point prevalence (from $0.08 \%$ up to $0.6 \%$ ) and overall or lifetime prevalence (in the range of 1 $2 \%$ ) values are likely to stay high or increase without surgical intervention because there are no data showing that conservative management can reduce them. Although the number of factors involved in the etiology of chronic wounds is extremely high, a recognition of functional gene variants significantly involved in wound healing and venous ulcer establishment, may help our prognosis, diagnosis and treatment capabilities of chronic wounds. Moreover, the characterization of known wound biomarkers, the identification of new biomarkers and the future development of new panels of biomarkers put the research approach at a good point for the prediction of chronic wound outcome, in conjunction to possible effective therapeutic potential.

In the same way, it is of great importance to identify and follow diagnostic-therapeutic protocols that provide for proper use of compression therapy and surgery. Currently there is a lack of clarity on contraindications, risk factors, adverse events and complications, when applying compression therapy for venous leg ulcer patients. Often guidelines are text heavy and not designed for practical clinical use. Patient focused clinical pathways may be a more practical way forward to optimize care for venous leg ulcer patient management.

Pharmacological therapy has shown that it can accelerate healing times and studies support the use of pentoxifylline, micronized purified flavonoid fraction and sulodexide. Some open questions remain about the duration of treatment and recurrence rates after pharmacological treatment.

\section{References}

1. Barwell JR, Deacon J, Harvey K, et al. Comparison of surgery and compression with compression alone in chronic venous ulceration (ESCHAR study): randomised controlled trial. Lancet 2004;363:1854-9.

2. Graham ID, Harrison MB, Nelson EA, et al. Prevalence of lower-limb ulceration: a systematic review of prevalence studies. Adv Skin Wound Care 2003;16:30516.

3. Nelzén O, Bergqvist D, Lindhagen A. Venous and non-venous leg ulcers: clinical history and appearance in a population study. Br J Surg 1994;81:182-7.

4. Nelzén O. Prevalence of venous leg ulcer; the importance of the data collection method. Phlebolymphol 2008; 15 : 143-50.

5. Rabe E, Pannier-Fischer F, Bromen K, et al. Bonner Venenstudie der Deutschen Gesellshaft für Phlebologie. Phlebologie 2003;32:1-14

6. Evans CJ, Fowkes FGR, Ruckley CV, Lee AJ. Prevalence of varicose veins and chronic venous insufficiency in men and women in the general population: Edinburgh Vein Study. J Epidemiol Community Health 1999;53: 149-53.

7. O'Donnell TF, Passman MA, Marston WA, et al. Management of venous leg ulcers: clinical practice guidelines of the Society for Vascular Surgery and the American Venous Forum. J Vasc Surg 2014;60:3S-59S.

8. Mosti G, De Maeseneer M, Cavezzi A, et al. Society for Vascular Surgery and American Venous Forum Guidelines on the management of venous leg ulcers: the point of view of the International Union of Phlebology. Int Angiol 2015;34:202-18.

9. Webb R (ed). Management of patients with venous leg ulcers. Challenges and current best practice. J Wound Care 2016; 25, EWMA document. Available from: https://ewma.org/fileadmin/ user_upload/EWMA.org/Project Portfol io/EWMA_Documents/Management_of patients_with_venous_leg_ulcers_FIN AL 2016.pdf

10. Gohel MS, Heatley F, Xinxue L, et al; for the EVRA trial investigators. A randomized trial of early endovenous ablation in venous ulceration. N Eng J Med 2018;378:2105-14.

11. Forssgren A, Fransson I, Nelzén O. Leg ulcer point prevalence can be decreased by broad scale intervention - a follow-up cross-sectional study of a defined geographical population. Acta Derm
Venereol 2008;88:252-6.

12. Forssgren A, Nelzén O. Changes in the aetiological spectrum of leg ulcers after a broad-scale intervention in a defined geographical population in Sweden. Eur J Vasc Endovasc Surg 2012:44:498-503.

13. Bonita R, Beaglehole R, Kjellström T. Basic epidemiology. 2nd ed. Geneva: World Health Organization; 2006.

14. Zamboni P. The big idea: iron-dependent inflammation in venous disease and proposed parallels in multiple sclerosis. J R Soc Med 2006;99:589-93.

15. Zamboni P, Izzo M, Tognazzo S, et al. The overlapping of local iron overload and HFE mutation in venous leg ulcer pathogenesis. Free Radic Biol Med 2006;40:1869-73.

16. Yan C, Boyd DD. Regulation of matrix metalloproteinase gene expression. J Cell Physiol 2006;211:19-26.

17. Zamboni P, Scapoli G, Lanzara V, et al. Serum iron and maxtrix metalloproteinase 9 variations in limbs affected by chronic venous disease and venous leg ulcers. Dermatol Surg 2005;31:19-22.

18. Gemmati D, Tognazzo S, Serino ML, et al. Factor XIII V34L polymorphism modulates the risk of chronic venous leg ulcer progression and extension. Wound Repair Regen 2004;12:512-7.

19. Gemmati D, Tognazzo S, Catozzi L, et al. Influence of gene polymorphisms in ulcer healing process after superficial venous surgery. J Vasc Surg 2006;44: 554-62.

20. Tognazzo S, Gemmati D, Palazzo A, et al. Prognostic role of factor XIII gene variants in nonhealing venous leg ulcers. J Vasc Surg 2006;44:815-9.

21. Herouy Y, Mellios P, Banderir E, et al. Inflammation in stasis dermatitis upregulates MMP-1, MMP-2 and MMP-3 expression. J Dermat Science 2001;25: 198-205.

22. Herouy Y, May AE, Pornschelegel G. et al. Lipodermatosclerosis is charaterized by elevated expression and activation of matrix mettalloproteinases: implications for venous ulcer formation. J Invest Dermatol 1998;111:822-7.

23. Zamboni P, De Mattei M, Ongaro A, et al. Factor XIII contrasts the effects of metalloproteinases in human dermal fibroblast cultured cells. Vasc Endovascular Surg 2004;38:413-38.

24. Ackerman Z, Seidenbaum M, Loewenthal E, Rubinow A. Overload of iron in the skin of patients with varicose ulcers. Possible contributing role of iron accumulation in progression of the disease. Arch Dermatol 1988;124:1376-8.

25. Raffetto JD. Dermal pathology, cellular biology, and inflammation in chronic 
venous disease. Thromb Res 2009;123: 66-71.

26. Zamboni P, Tognazzo S, Izzo M, et al. Hemochromatosis $\mathrm{C} 282 \mathrm{Y}$ gene mutation increases the risk of venous leg ulceration. J Vasc Surg 2005;42:309-14.

27. Gemmati D, Federici F, Catozzi L, et al. DNA-array of gene variants in venous leg ulcers: detection of prognostic indicators. J Vasc Surg 2009;50:1444-51.

28. Mannello F, Ligi D, Canale M, Raffetto JD. Omics profiles in chronic venous ulcer wound fluid: innovative applications for translational medicine. Expert Rev Mol Diagn 2014; 14:737-62.

29. Ligi D, Mosti G, Croce L, et al. Chronic venous disease - Part II: Proteolytic biomarkers in wound healing. Biochim Biophys Acta 2016;1862:1900-8.

30. Raffetto JD, Mosti G, Santi M, et al. Matrix metalloproteinase profiles in chronic venous ulcer wound fluid of inflammatory and granulating venous leg ulcers. J Vasc Surg Venous Lymphat Disord 2015;3:119-20.

31. Mannello F, Raffetto JD. Matrix metalloproteinase activity and glycosaminoglycans in chronic venous disease: the linkage among cell biology, pathology and translational research. Am J Transl Res 2011;3:149-58.

32. Ligi D, Mosti G, Croce L, et al. Chronic venous disease - Part I: Inflammatory biomarkers in wound healing. Biochim Biophys Acta 2016;1862:1964-74.

33. Raffetto JD, Eberhardt RT, Dean SM, et al. Pharmacologic treatment to improve venous leg ulcer healing. J Vasc Surg Venous Lymphat Disord 2016;4:371-4.

34. Eberhardt RT, Raffetto JD. Chronic venous insufficiency. Circulation 2014; 130:333-46.

35. O'Donnell TF, Passman MA, Marston WA, et al. Management of venous leg ulcers; clinical practice guidelines of the society for vascular surgery and the American venous forum. J Vasc Surg 2014;60:3S-59S.

36. Tang JC, Marston WA, Kirsner RS. Wound Healing Society (WHS) venous ulcer treatment guidelines: what's new in five years? Wound Repair Regen 2012;20:619-37.

37. Weller C, Evans S. Venous ulcer management in general practice - practice nurses and evidence based guidelines. Aust Fam Physician 2012;41:331-7.

38. Boehler K, Kittler S, Stolkovich S, Tzaneva T: Therapeutic Effect of Compression Stockings

Versus no Compression on Isolated Superficial Vein Thrombosis of the Legs: A Randomized Clinical Trial. Eur J Vasc
Endovasc Surg 2014;48:465-71.

39. EWMA. Understanding compression therapy. EWMA Position Document; 2003. Available from: https://ewma.org/ fileadmin/user_upload/EWMA.org/Posit ion_documents_2002-2008/ Compression.pdf

40. Van Hecke A, Grypdonck M, Defloor T. Guidelines for the management of venous leg ulcers: a gap analysis J Evan Clin Pract 2008;14:812-22.

41. Hopkins A, Bull R, Worboys F. Needing more: the case for extra high compression for tall men in UK leg ulcer management. Veins and Lymphatics 2017;6:6630.

42. Wu SC, Crews RT, Skratsky M, et al. Control of lower extremity edema in patients with diabetes: double blind randomized controlled trial assessing the efficacy of mild compression diabetic socks. Veins and Lymphatics 2017;6: 6637.

43. European Dermatology Forum. S3 guideline for diagnostics and treatment of venous leg ulcers. EDF guidelines leg ulcers, version 4.0; 2014. Available from: http://turkderm.org.tr/turkderm Data/Uploads/files/S3-Guideline \%2 0on\%20venous\%20leg\%20ulcers\%20$\% 20$ Update\%202014.pdf

44. Rabe E, Partsch H, Junger $M$, et al. Guideline for clinical studies with compression devices in patients with venous disorders. Eur J Vasc Endovasc Surg 2008;35:494-500.

45. Wittens C, Davies AH, Bækgaard N, et al. Cliniwcal Practice Guidelines of the European Society for Vascular Surgery (ESVS). Eur J Vasc Endovasc Surg 2015;49:678-737.

46. Neumann HAM, Junger M, Munte K, Rabe E et al: European Dermatology Forum guideline for diagnostic and treatment of venous leg ulcers. J Eur Acad Dermatol Venereol 2016;30:1843-75.

47. Assenheimer B, Augustin M, Braunwarth $\mathrm{H}$, et al. Consensus recommendations for compression therapy for patients with venous ulcers. J Eur Acad Dermatol Venereol 2013;13:41.

48. Kimmel HM, Robin AL. An evidencebased algorithm for treating venous leg ulcers. Cochrane Database Syst Rev Wounds 2013:25;242-50.

49. Muller R. Pentoxifylline: biomedical profile. J Med 1979;10:307-29.

50. Jull AB, Arroll B, Parag V, Waters J. Pentoxifylline for treating venous leg ulcers. Cochrane Database Syst Rev 2012;12:CD001733.

51. Shoab SS, Porter J, Scurr JH, et al. Endothelial activation response to oral micronized flavonoid therapy in patients with chronic venous disease - a prospective study. Eur J Vasc Endovasc Surg 1999; 17:313-8.

52. Smith PD. Micronized purified flavonoid fraction and the treatment of chronic venous insufficiency: microcirculatory mechanisms. Microcirculation 2000;7:S35-40.

53. Coleridge-Smith P, Lock C, Ramelet AA. Venous leg ulcer: a metaanalysis of adjunctive therapy with micronized purified flavonoid fraction. Eur J Vasc Endovasc Surg 2005;30:198-208.

54. Coccheri S, Scondotto G, Agnelli G, et al. Venous armo $f$ the SUAVIS Group: randomized, double-blind, multicentre, placebo-controlled study of Sulodexide in the treatment of venous leg ulcers. Thromb Haemost 2002;87:947-52.

55. Mannello F, Ligi D, Raffetto JD. Glycosaminoglycan sulodexide modulates inflammatory pathways in chronic venous disease. Int Angiol 2014;33: 23642.

56. Nelson EA. Venous leg ulcers. BMJ Clin Evid 2011;1902.

57. Weller C, Barker A, Darby I, et al. Aspirin in venous leg ulcer study (ASPiVLU): study protocol for a randomised controlled trial. Trials 2016; 17:192.

58. Nelson EA, Adderley U. Venous leg ulcers. BMJ Clin Evid 2016:1902.

59. Varatharaian L, Thapar A, Lane T, et al. Pharmacological adjuncts for chronic venous ulcer healing: a systematic review. Phlebology 2016;31:356-65.

60. Gonzalez Ochoa A. Sulodexide and phlebotonics in the treatment of venous ulcer. Int Angiol 2016;36:82-7.

61. Wu B, Lu J, Yang M, Xu T. Sulodexide for treating venous leg ulcers. Cochrane database Syst Rev 2016;6:CD010694.

62. Tessari M, Gianesini S, Spath P, et al. A novel concept of pneumatic pump in the outpatient management of mixed leg ulceration: a pilot study. Veins and Lymphatics 2016;5:5266.

63. Martinez-Zapata MJ, Vernooij RW, Uriona Tuma SM, et al. Phlebotonics for venous insufficiency. Cochrane Database Syst Rev 2016;4:CD003229.

64. Parker CN, Finlayson KJ, Edwards HE. Predicting the likelihood of delayed venous leg ulcer healing and recurrence: development and reliability testing of risk assessment tools. Ostomy Wound Manag 2017;63:16-33.

65. Norman G. Dressings and topical agents for treating venous leg ulcers. Cochrane Database Syst Rev 2018;2018: CD012583. 